\title{
The Effects of Statin Treatment on Outcomes following Traumatic Brain Injury
}

John Whyte ${ }^{1}$, Jessica M. Ketchum ${ }^{2,3}$, Jenny Bogner ${ }^{4}$, Robert C. Brunner ${ }^{5}$, Flora M. Hammond ${ }^{6}$, Ross Zafonte ${ }^{7}$, Gale G. Whiteneck ${ }^{2}$, Alan Weintraub ${ }^{8}$

1. Moss Rehabilitation Research Institute, Einstein Healthcare Network, Elkins Park, PA, USA

2. Research Department, Craig Hospital, Englewood, CO;

3. Traumatic Brain Injury Model Systems National Data and Statistical Center, Englewood, CO.

4. Ohio State University Wexner Medical Center, Columbus, Ohio

5. University of Alabama at Birmingham, Spain Rehabilitation Center, Birmingham, Alabama

6. Indiana University School of Medicine; Rehabilitation Hospital of Indiana, Indianapolis, Indiana

7. Department of Physical Medicine and Rehabilitation, Harvard Medical School; Spaulding Rehabilitation Hospital; Massachusetts General Hospital; Brigham and Women's Hospital; \& Home Base, Boston, Massachusetts, USA

8. Craig Hospital, Rocky Mountain Regional Brain Injury System, Englewood, CO

\author{
Corresponding Author: \\ John Whyte, MD, PhD \\ Moss Rehabilitation Research Institute \\ 50 Township Line Road \\ Elkins Park PA 19027 \\ (215) 663-6872 \\ jwhyte@einstein.edu
}

This is the author's manuscript of the article published in final edited form as:

Whyte, J., Ketchum, J. M., Bogner, J., Brunner, R. C., Hammond, F. M., Zafonte, R., ... Weintraub, A. (2018). Effects of Statin Treatment on Outcomes after Traumatic Brain Injury. Journal of Neurotrauma, 36(1), 118-125. https://doi.org/10.1089/neu.2017.5545 
The contents of this manuscript were developed under grants from the National Institute on Disability, Independent Living, and Rehabilitation Research to the Moss Traumatic Brain Injury Model System at Moss Rehabilitation Research Institute (\#90DP0037-02-01), the Rocky Mountain Regional Brain Injury System at Craig Hospital (\#90DP0034-01-00), the Ohio Regional Traumatic Brain Injury Model System at Wexner Medical Center (90DP0040), the UAB Traumatic Brain Injury Model Systems at the University of Alabama (\#90DP0044), the Traumatic Brain Injury Model System at Indiana University School of Medicine and Rehabilitation Hospital of Indiana (\#90DP0036-01-00), the Spaulding-Harvard Traumatic Brain Injury Model System at Spaulding Rehabilitation Hospital (\#90DP0039-03-01), and the Traumatic Brain Injury Model Systems National Data and Statistical Center at Craig Hospital (\#90DP0084-01-00). NIDILRR is a Center within the Administration for Community Living (ACL), Department of Health and Human Services (HHS). The contents of this manuscript do not necessarily represent the policy of NIDILRR, ACL, or HHS, and you should not assume endorsement by the Federal Government.

Acknowledgements: The authors would like to thank Dwight Henderson, Health Care Systems, Inc., for providing the pharmacy search services during the planning and data collection stages of this study, and Prathap Jacob Joseph, UT Health McGovern Medical School for participation in the pilot phase of this work. We would also like to thank David Mellick for database design and management, and Aash Bhandari, Sharon Guin-Renfroe, Kara Jodry, Devon Kratchman, Mike Mahaffey, Angela M. Philippus, Rebecca Runkel, Krista Smith, Hannah Thompson, and Beth Windisch for assistance with data collection. Finally, Mary Czerniak is gratefully acknowledged for assistance in preparing the manuscript.

Running Head: Statins and TBI Outcome

Key Words: Brain injuries, Neuroprotection, Hydroxymethylglutaryl-CoA reductase inhibitors, Treatment outcome, Propensity score 


\section{Abstract}

Neuroprotective treatments that have shown promise in reducing secondary injury and improving recovery in animal models of traumatic brain injury, have not been found effective to date in humans. One reason may be the delay after injury in initiating treatment. Statin medications are among the promising neuroprotective agents in animal models, and their presence in the bloodstream of many individuals at the time of injury, might optimize their clinical impact.

This observational study conducted by a subset of centers participating in the NIDILRRfunded TBI Model System program sought to examine the effects of being on statin medication at the time injury, on functional outcomes. Participants over the age of 50 were prospectively enrolled inpatient rehabilitation admission. Demographic data, cardiovascular history, and brain injury history were obtained through chart abstraction and interview. Prescription medication use in the year prior to enrollment was determined from a national pharmacy search service. Propensity scoring was used to create 49 pairs of participants who were well matched on demographic and clinical attributes but discordant for statin use.

The treated and untreated participants did not differ on initial Glasgow Coma Score, time until commands were followed, duration of post-traumatic amnesia, or FIM scores at rehabilitation admission, discharge, or 1 year post-injury, nor on acute or rehabilitation hospital lengths of stay. Evidence of greater and lesser statin compliance was not associated with outcome. This study did not provide support for a clinically important benefit of statin use at the time of moderate to severe TBI. 
$\underline{\text { Introduction }}$

Statins, also known as 3-hydroxy-3-methylglutaryl co-enzyme A (HMG-CoA) reductase inhibitors, are medications commonly used in the treatment of elevated cholesterol, and are universally recognized for their effectiveness in reducing morbidity and mortality associated with coronary heart disease by lowering serum cholesterol levels ${ }^{1,2}$. Spearheaded by unexpected findings of a reduced incidence of stroke in the first major statin trials conducted in patients with coronary heart disease ${ }^{3,4}$, the association between statin use and reduced incidence of ischemic stroke has since been consistently demonstrated in the literature ${ }^{1,5}$. While the exact mechanism of reduced stroke incidence is unclear, it is assumed that this benefit is driven by statins' reduction of low-density lipoprotein-cholesterol (LDL-C $)^{5}$.

Over and above their influence on attenuated stroke risk, recent data suggest that statins exhibit a number of neuroprotective properties, including anti-inflammation, antioxidation, endothelial protection, antiapoptotic effects, increased angiogenesis, and enhance neurogenesis and synaptogenesis ${ }^{6-9}$. Statins have also been shown to inhibit the expression of aquaporin-4 in an animal model of subarachnoid hemorrhage, ${ }^{10}$ and to reduce the incidence of vasospasm and possibly improve outcome after subarachnoid hemorrhage in humans. ${ }^{11,12}$ In addition, statins have demonstrated positive effects on the incidence of neurodegenerative diseases including Alzheimer's disease ${ }^{13,14}$ and Parkinson's disease ${ }^{15-17}$. Furthermore, the medication class has demonstrated positive treatment effects in animal models of multiple sclerosis $^{18-21}$ and brain tumors ${ }^{22}$. Guided by these findings, within the last decade a number of researchers have begun investigating the effects of statins on traumatic brain injury (TBI). In animal models, statins reduced contusion volume and hippocampal neuronal loss, improved rotarod and Morris water maze performance, and enhanced neurogenesis ${ }^{23-26}$.

The context of the current research is the repeated failure to identify an effective neuroprotective agent that can reduce the ultimate severity of human TBI and the many clinical 
sequelae that result. Much attention has been devoted to the sources of this failure, but one important factor may be the chronology: animal trials are able to administer the neuroprotective agent immediately after (or sometimes even before) the injury. ${ }^{27}$ If statin drugs have neuroprotective effects, they would be one of the few strategies likely to be implemented prior to the trauma.

With respect to clinical translation, while the number of published studies is limited, the effect of statins on TBI in humans has been less robust. Robertson et al. ${ }^{9}$ demonstrated that administration of atorvastatin for 7 days post-injury was safe, but did not find any significant differences in neurological recovery after mild TBI, when compared to a placebo group. In contrast, Sánchez-Aguilar demonstrated that rosuvastatin use was associated with a reduction in disability scores among individuals with moderate-severe TBI, indicating that statins may promote neurological recovery ${ }^{28}$. Likewise, by administering rosuvastatin to adults with a history of TBI, Tapia-Perez found that statins were associated with a reduction of amnesia time, further lending evidence to benefits in functional outcomes ${ }^{29}$. In sum, while evidence for the efficacy of statin medications for TBI in humans is inconsistent, prior research demonstrates potential for the drugs to improve outcomes following brain injury.

This study was performed as part of the TBI Model System research program, funded by the National Institute on Disability, Independent Living, and Rehabilitation Research (NIDILRR), and conducted by a subset of 6 Model System institutions. The analysis combined data collected specifically for this study with data collected prospectively as part of the longitudinal Model System database effort. We sought to apply a propensity score analysis method to compare a range of clinical outcomes in comparable patients with and without statin prescriptions. Our aims were to assess the impact of statin use on a range of clinical outcomes ranging from acute to subacute, and to assess whether any benefits of statin use that were found were related to estimates of medication compliance. 


\section{Materials and Methods}

\section{Participants}

Individuals were considered for participation in this study if they were enrolled in the Traumatic Brain Injury Model System (TBIMS) and were at least 50 years old (the prevalence of statin use at younger ages is low, as determined in a prior feasibility pilot study conducted by this investigator group). In brief, TBIMS eligibility requires an injury to the brain caused by an external physical force, of at least a complicated mild nature, as defined by at least one of the following: positive clinical imaging, Glasgow Coma Score $<13$, duration of unconsciousness $>$ 30 minutes, or duration of post-traumatic amnesia $>24$ hours. Eligibility also requires age $>15$ and presentation to a participating hospital within 72 hours of injury. The full details on Model System eligibility can be found at https://www.tbindsc.org. Eligibility for this study additionally required that the participant be free of significant neurologic disability prior to the TBI. This study was IRB approved at all sites and informed consent for study participation was obtained from each participant or a legally authorized representative. Because a national pharmacy search strategy was used for collecting statin use data, individuals were excluded post-consent if a valid pharmacy search could not be obtained.

\section{Measures}

The majority of data collected for this study was obtained from the TBIMS longitudinal database, and included demographic, injury, and clinical outcome data. These were supplemented by data on statin prescription and use, and by additional data on comorbid cardiovascular conditions.

Demographic data included age at injury, gender, body mass index, education level (high school or greater vs. less than high school), employment status (employed/not employed) at the time of injury, and race/ethnicity (white/not Hispanic vs. non-white/Hispanic). We also 
categorized the mechanism of injury (fall/vehicular/other), and the presence of a range of preinjury functional limitations. Comorbid conditions that were thought to be relevant to the prescription of statins or were likely independent sources of disability were also collected. Conditions that might independently contribute to disability included chronic obstructive pulmonary disease, diabetes, liver disease, and tobacco and problematic substance use.

National pharmacy searches were used to determine statin use and adherence and were performed by Health Care Systems, Inc., Montgomery, AL.

The following clinical measures were collected prospectively under the standard TBIMS longitudinal data collection protocol:

- Glasgow Coma Score collected in the emergency department;

- Time to Follow Commands (TFC), defined as the number of days between the TBI and the first date that the individual was able to follow simple motor commands accurately at least two times consecutively in a 24-hour period;

- Duration of post-traumatic amnesia (PTA), calculated as the number of days between the TBI and the first of two occasions within 72 hours that the participant was fully oriented. Full orientation was defined as a score above 25 on the Orientation Log, ${ }^{30}$ or documentation of consistent orientation for 72 hours in the acute medical record (i.e., prior to rehabilitation admission);

- Motor FIM and Cognitive FIM at the time of admission to acute inpatient rehabilitation, at rehabilitation discharge, and at 1-year post-injury;

- Acute care length of stay; and

- Inpatient rehabilitation length of stay.

Whereas the first 3 of these measures are commonly thought of as indicators of the severity of $\mathrm{TBI}$, in this study we elected to treat them, along with the rest, as potential clinical outcomes 
because all 3 are measured after the "administration" of the study drug and there is no evidence available to assess how early a neuroprotective effect might appear. In addition, as noted, one prior study has suggested a possible impact of statins on duration of PTA.

\section{Procedure}

Demographic and injury data were collected through a combination of chart review and patient or caregiver interview. History of comorbid cardiovascular conditions was determined from chart review supplemented by a more detailed standardized interview of the participant or caregiver. For each condition, the participant was asked whether they had ever been diagnosed with that condition and whether it was functionally limiting.

GCS scores were obtained through review of emergency department records, with concurrent review for the administration of sedatives or paralytic agents. These, coupled with intubation, led to a high rate of missing GCS data. Time until commands were followed and duration of PTA were determined by chart review for those who were already following commands and/or fully oriented on rehabilitation admission. Those who were still unconscious or disoriented were assessed prospectively for the emergence of command following and for resolution of PTA. Patients who were still in PTA at the time of rehabilitation discharge $(N=48$, approximately $16 \%$ of the total sample), were given an imputed duration to the day of discharge.

Participants' names and addresses were entered into the TBIMS National Data and Statistical Center's (NDSC) study database by local data collectors and sent electronically to the pharmacy search service on a periodic basis. The search produced prescription data for the year prior to the initiation of the search. This resulted in less than 1 year's data and variations in the time covered by the search depending on the lag between injury and search initiation. Searches were returned via the NDSC database and could report no prescriptions or a list of prescriptions, some of which were associated with prescriber identifying information. Local data 
collectors sought to verify the validity of these searches by interviewing the participant or caregiver in person or by telephone. Negative searches were considered valid if the participant reported that s/he had not been taking any prescribed medications in the year prior to injury. Positive searches were considered valid if the participant recognized some, but not necessarily all, prescriptions or prescribers. Failure to recognize individual medications or prescribers alone was not considered evidence of an invalid search. Participants with invalid searches (whether those searches produced prescriptions or not) were excluded from further analysis. Valid pharmacy searches were examined for presence of any statin prescription (from a standard list of drugs in this class), and the date, strength, daily dosage, and number of pills supplied in each prescription. For the primary analysis, participants were considered to have been prescribed statins if a valid pharmacy search contained 1 or more statin prescriptions in the year prior to injury.

In the event that statin use was associated with clinical benefit, we hoped to determine whether this benefit was greater in individuals that were more adherent to their statin prescriptions. Various methods have been used to quantify adherence from pharmacy search records. ${ }^{31}$ However, in many studies of chronic diseases such as hypertension, clinical outcomes are likely driven by average adherence over time. In contrast, we assumed that adherence in the days preceding injury was most relevant to a neuroprotective effect. Accordingly, for each statin user, we determined whether the number of statin pills that had been dispensed prior to injury was sufficient to allow for continuous adherence to the time of injury or was not. 


\section{Data Analysis}

SAS v.9.4. ${ }^{1}$ was used for all data analysis and a significance level of $5 \%$ was used for all tests. A total of 295 patients consented to participate in the study. History of statin use was available for 224 patients and missing due to invalid searches for 71 patients $(24.1 \%)$. Of the 224 patients whose statin history was available, 69 patients were on statins pre-injury (30.8\%) and 155 were not $(69.2 \%)$. Patient characteristics were summarized separately for those with and without a history of pre-injury statin use using frequency counts and percentages for nominal variables and means and standard deviations (SD) for continuous variables.

Differences in measured characteristics between treated and untreated patients were assessed using two methods as described in Austin (2009). ${ }^{32}$ First, the statistical significance of either the difference in the proportion of patients having a dichotomous risk factor or the difference in the mean of a continuous covariate between treated and untreated patients was tested. A chi-square test was used for dichotomous variables and a $t$-test was used for continuous variables. Second, the standardized difference was computed for each covariate. The standardized differences are defined as

$$
d=\frac{100\left(\bar{x}_{T}-\bar{x}_{C}\right)}{\sqrt{\left(s_{T}^{2}+s_{C}^{2}\right) / 2}} \quad \text { for continuous variables }
$$

and

$$
d=\frac{100\left(p_{T}-p_{C}\right)}{\sqrt{\left[p_{T}\left(1-p_{T}\right)+p_{C}\left(1-p_{C}\right)\right] / 2}} \quad \text { for dichotomous variables. }
$$

\footnotetext{
${ }^{1}$ Copyright (c) 2002-2012 by SAS Institute Inc., Cary, NC, USA.
} 
Unlike the $t$-test and chi-square test, the standardized difference is not influenced by sample size and thus can be used to compare balance in variables between unmatched groups of unequal sample sizes. Furthermore, it can be used to compare the relative balance across measures with different units. It has been suggested that a standardized difference of greater than 10 percent represents meaningful imbalance in a given covariate between treatment groups. ${ }^{33}$ However the threshold for meaningful imbalance likely depends on the prognostic value of the covariate. ${ }^{34}$

The focus of this study was to assess the impact of being on a statin at the time of injury on clinical indicators of TBI severity and functional status. Thus, we sought to balance on a range of potential confounders of the clinical outcomes of interest, including age, BMI, gender, race/ethnicity, employment history, level of education, cause of injury, history of problematic alcohol or substance use, tobacco use, pre-injury limitations, and other health conditions. A number of cardiovascular diagnoses were very strong predictors of statin use such that few participants with those cardiovascular diagnoses but not on statins were available for matching. Certain cardiac conditions were considered as likely confounders by their mere presence (e.g., congestive heart failure), whereas others (e.g., having had angioplasty) were not identified as confounders unless they were associated with functional limitations. Due to the low numbers of individual comorbidities, some of these were collapsed into larger categories for analysis, as noted in the table legend. The frequency distribution and the grouping of these variables for the purpose of analysis are shown in Table 1.

\section{\{Table 1 about here $\}$}

A propensity score model was developed to predict the probability of being on statins prior to TBI. A structured, iterative approach, similar to that of Rosenbaum and Rubin, ${ }^{35}$ was used to balance the aforementioned measured covariates. More specifically, a propensity score 
model was developed to balance the distribution of these confounders between treated and untreated patients within each quintile of the estimated propensity score (age and BMI were included as quadratic terms). A greedy matching algorithm was then used to match subjects using calipers that were defined to have a maximum width of 0.2 standard deviations of the logit of the estimated propensity score. The degree to which matching on the propensity score created a matched sample was then assessed using the same methodology as the unmatched sample (statistical testing and standardized differences).

The distribution of the differences in outcomes between the treated and untreated groups in the matched sample was examined visually using normal quantile plots and ShapiroWilks tests to assess for normality. The difference in outcomes between the treatment and control groups was compared using paired $t$-tests for normally distributed differences and Wilcoxon signed-rank tests for non-normally distributed differences.

Within the group of 69 subjects on statins, outcome variables were further compared between adherent and non-adherent subjects using independent two sample t-tests for normally distributed outcomes and Wilcoxon rank-sum tests for non-normally distributed outcomes.

\section{$\underline{\text { Results }}$}

The distribution of the potential covariates is summarized for statin users and non-users in the entire sample, prior to the matching procedure, in the left side of Table 2 . There are a number of variables where the differences between statin users and non-users were statistically significant including age at injury, employment status, cause of injury, and other health conditions. Furthermore, all covariates considered, except education, demonstrated at least moderate imbalance with standardized differences $>0.1$. The propensity score matching algorithm produced 49 matched pairs and the distribution of the covariates after matching is summarized 
in the right side of Table 2. The matched sample demonstrated acceptable balance in nearly all measured covariates as indicated by insignificant $p$-values (all >0.52) and small standardized differences (all < 0.13). While some covariates still showed "moderate" imbalance after matching (gender, cause of injury, problematic substance use), the standardized differences were close to the threshold of 0.1 , and none was considered a particularly strong prognostic factor. Figure 1 illustrates the area of common support by quartile of the propensity scores, suggesting that the groups were well matched over the first 3 propensity quartiles and showed only moderate imbalance in the highest quartile.

$\{$ Table 2 about here $\}$

\{Figure 1 about here\}

Using the matched sample, clinical outcomes were then compared between statin users and non-users. All outcomes are summarized by group using means/standard deviations (SD) and medians/interquartile ranges (IQRs) in Table 3 along with differences in outcomes between the groups. The medians and IQRs are preferred over the means and SDs as descriptors of central tendency and spread when data are markedly skewed and not normally distributed. Statistical significance of differences was assessed with a paired $t$-test or a Wilcoxon signed-rank test depending on the results of the Shapiro-Wilks test of normality. Note that the number of pairs available for analysis was reduced for the GCS (because of paralysis and intubation) and the 1year FIM outcomes (because the 1-year mark had not been reached for all participants). As can be seen in the table, none of the variables assessed demonstrated a significant benefit of statin use. Moreover, the differences between groups were small and inconsistent in direction. Because the slight remaining imbalance in gender had the greatest potential to bias the results, we ran parallel mixed model analyses with raw and with log-transformed outcome scores, in 
which gender was added as a covariate. The pattern of results was similar in these analyses (data not shown).

\{Table 3 about here $\}$

As noted, 69 participants were prescribed statins prior to injury, not all of whom could be matched with non-users for the primary analysis. However, the influence of statin adherence within this complete sample of 69 users was assessed by comparing the same set of clinical outcomes between those with a high probability of adherence $(54 ; 78.3 \%)$ to those who were judged non-adherent (15; $21.7 \%)$, as shown in Table 4. Being presumed adherent to prescribed statin doses was not significantly associated with better clinical outcomes. Only one measureCognitive FIM at rehabilitation discharge - showed marginal significance with a trend toward benefit.

\{Table 4 about here $\}$

Given these negative results, it is important to assess the study's statistical power to detect a neuroprotective effect of statins. A sample size of 49 pairs has $80 \%$ power to detect an effect size of at least 0.41 with a paired $t$-test and of at least 0.33 with a non-parametric Wilcoxon signed-ranks test, which is sufficient sample to detect a small to medium treatment effect. Taking into account missing data and not considering the GCS measures, a sample size of 37 pairs has $80 \%$ power to detect an effect size of at least 0.47 with a paired $t$-test and of at least 0.38 with a non-parametric Wilcoxon signed-rank test The measured effect sizes of statin use on the various outcomes in this study ranged between 0 and .33 (mean difference/standard deviation), which are considered small or negligible; thus, lack of statistical significance in this study is likely not attributable simply to insufficient power. 


\section{Discussion}

The results of this study do not provide evidence that use of a statin drug at the time of a moderate to severe traumatic brain injury improves functional outcomes in the post-acute period, as assessed by a range of indicators measured from the first hours post-injury through the time of rehabilitation hospital discharge and at one year. Moreover, those likely to be adherent in statin treatment did not appear to differ in their outcomes from those less likely to be adherent. Although the size of the sample studied cannot rule out a small effect size, there was not a consistent trend favoring statin use across the outcomes evaluated.

In interpreting these study findings, several limitations should be considered. This study only included individuals who were initially treated at an acute care hospital and eventually transferred to acute inpatient rehabilitation. That is, this study does not represent individuals who never received inpatient rehabilitation (e.g., those who are discharged from acute care to home, a non-TBIMS long-term acute care hospital, or skilled nursing facility). This study also does not represent younger individuals with TBI as inclusion was restricted to age 50 years or older. However, the study sample is relevant to the population of older individuals likely to already be on statins at the time of injury, since this is relatively uncommon at younger ages. Despite the sizeable sample of participants enrolled, nearly a quarter were lost because of invalid pharmacy searches. These likely resulted from obtaining prescriptions from smaller nonparticipating pharmacies and in a few cases, the Veterans Administration pharmacies. Moreover, even when we have valid prescription data, the fact that a participant recently refilled a statin prescription is only indirect evidence of consuming the statin medication regularly in the days leading up to injury. Moreover, our comparison of probable adherent and non-adherent statin users was not propensity adjusted, so these subgroups may have differed in other ways. However, if such a bias existed one would predict better outcomes in the kinds of patients likely to be treatment adherent, but this was not found. In addition, participants did not continue 
previously prescribed statins in the early days after injury when they received only parenteral medications. Thus, to the extent that any neuroprotective effect might depend, not primarily on the presence of statins at the moment of injury, but on continued treatment in the early days after injury, interruption of statin treatment in the ICU might have masked the drug's benefits.

Our study did not examine the effects of statins received at later points post-injury, nor did we have statin prescription data for a full year prior to injury. However, a substantial effect of statin use at later points in the absence of any acute effect appears unlikely given the drug's known mechanisms of action, and the compliance data that appears most relevant to the effect on injury is recent data, not year-old data. Our study was also unable to assess the possibility of a neuroprotective benefit from one or more specific statin agents, because of the size of the sample.

Finally, our results are limited by the propensity score method itself. We were able to match less than $50 \%$ of the original sample in the final data analysis. While the matched sample and the original sample were similar on most covariates, some were notably divergent (e.g. there was a higher percentage of females among non-statin users in the original sample, see Table 2). After matching there were still minor imbalances that could have obscured statin effects, though additional adjustment for gender did not alter the results. We limited our variables to those factors known before injury, to avoid using any predictors that might already be affected by the drug. However, this prevented us from making use of 3 commonly used "injury severity indicators": the GCS, time until commands are first followed, and duration of post-traumatic amnesia. Moreover, we were not able to seek balance on the risk factors that drive statin prescribing both because we did not have access to some (e.g., cholesterol level), and because others (e.g., previous heart attack) were so strongly associated with statin use that individuals with those indicators who were not on statins were exceedingly uncommon. In 
addition, the remaining variables used in the propensity score were much improved but not completely balanced.

\section{$\underline{\text { Conclusion }}$}

This observational study comparing individuals receiving and not-receiving statin drugs at the time of a severe traumatic brain injury failed to find evidence of a neuroprotective effect of statins, nor did a comparison of those likely to be adherent vs. non-adherent to statin prescription demonstrate outcome differences. The study design could not rule out a small beneficial effect of statins but the directional trends among different outcome measures were inconsistent. 


\section{$\underline{\text { References }}$}

1. Law, M.R., Wald, N.J., and Rudnicka, A. (2003). Quantifying effect of statins on low density lipoprotein cholesterol, ischaemic heart disease, and stroke: systematic review and meta-analysis. BMJ 326, 1423.

2. Tobert, J.A. (2003). Lovastatin and beyond: the history of the HMG-CoA reductase inhibitors. Nat Rev Drug Disc 2, 517-526.

3. (1994). Randomised trial of cholesterol lowering in 4444 patients with coronary heart disease: the Scandinavian Simvastatin Survival Study Group (4S). The Lancet 344, 1383-1389.

4. Sacks, F.M., Pfeffer, M.A., Moye, L.A., Rouleau, J.L., Rutherford, J.D., Cole, T.G., Brown, L., Warnica, J.W., Arnold, J.M.O., Wun, C.C., Davis, B.R., and Braunwald, E. (1996). The effect of pravastatin on coronary events after myocardial infarction in patients with average cholesterol levels. New Engl J Med 335, 1001-1009.

5. Amarenco, P., Labreuche, J., Lavallée, P., and Touboul, P.J. (2004). Statins in stroke prevention and carotid atherosclerosis. Stroke 35, 2902-2909.

6. Vaughan, C.J., and Delanty, N. (1999). Neuroprotective properties of statins in cerebral ischemia and stroke. Stroke 30, 1969-1973.

7. van der Most, P.J., Dolga, A.M., Nijholt, I.M., Luiten, P.G., and Eisel, U.L. (2009). Statins: mechanisms of neuroprotection. Prog in Neurobiol 88, 64-75.

8. Stêpieñ, K., Tomaszewski, M., and Czuczwar, S.A.J. (2005). Neuroprotective properties of statins. Pharm Rep 57, 561-569.

9. Robertson, C.S., McCarthy, J.J., Miller, E.R., Levin, H., McCauley, S.R., Swank, P.R., and et, a.I. (2017). Phase II clinical trial of Atorvastatin in mild traumatic brain injury $\mathrm{J}$ Neurotraum 34, 1394-1401. 
10. Chen, J.H., Yang, L.K., Chen, L., Wang, Y.H., Wu, Y., Jiang, B.J., Zhu, J., and Li, P.P. (2016). Atorvastatin ameliorates early brain injury after subarachnoid hemorrhage via inhibition of AQP4 expression in rabbits. Int J Mol Med 37, 1059-1066.

11. Shen, J., Huang, K.Y., Zhu, Y., Pan, J.W., Jiang, H., Weng, Y.X., and Zhan, R. (2017). Effect of statin treatment on vasospasm-related morbidity and functional outcome in patients with aneurysmal subarachnoid hemorrhage: a systematic review and metaanalysis. J Neurosurg 127, 291-301.

12. Lynch, J.R., Wang, H., McGirt, M.J., Floyd, J., Friedman, A.H., Coon, A.L., Blessing, R., Alexander, M.J., Graffagnino, C., Warner, D.S., and Laskowitz, D.T. (2005). Simvastatin reduces vasospasm after aneurysmal subarachnoid hemorrhage: results of a pilot randomized clinical trial. Stroke 36, 2024-2026.

13. Höglund, K., and Blennow, K. (2007). Effect of HMG-CoA Reductase Inhibitors on $\beta$ Amyloid Peptide Levels. CNS Drugs 21, 449-462.

14. Wolozin, B., Manger, J., Bryant, R., Cordy, J., Green, R.C., and McKee, A. (2006). Reassessing the relationship between cholesterol, statins and Alzheimer's disease. Acta Neurol Scand 114, 63-70.

15. Huang, X., Chen, H., Miller, W.C., Mailman, R.B., Woodard, J.L., Chen, P.C., Xiang, D., Murrow, R.W., Wang, Y.Z., and Poole, C. (2007). Lower low-density lipoprotein cholesterol levels are associated with parkinson's disease. Mov Disord 22, 377-381.

16. Wahner, A.D., Bronstein, J.M., Bordelon, Y.M., and Ritz, B. (2008). Statin use and the risk of Parkinson disease. Neurol 70, 1418-1422.

17. Wolozin, B., Wang, S.W., Li, N.C., Lee, A., Lee, T.A., and Kazis, L.E. (2007). Simvastatin is associated with a reduced incidence of dementia and Parkinson's disease. BMC medicine 5, 20.

18. Aktas, O., Waiczies, S., Smorodchenko, A., Dörr, J., Seeger, B., Prozorovski, T., Sallach, S., Endres, M., Brocke, S., Nitsch, R., and Zipp, F. (2003). Treatment of 
relapsing paralysis in experimental encephalomyelitis by targeting Th1 cells through atorvastatin. J Exp Med 197, 725-733.

19. Floris, S., Blezer, E.L.A., Schreibelt, G., Dopp, E., van der Pol, S.M.A., SchadeeEestrmans, I.L., Nicolay, K., Dijkstra, C.D., and de Vries, H.E. (2004). Blood-brain barrier permeability and monocyte infiltration in experimental allergic encephalomyelitis. Brain 127, 616-627.

20. Greenwood, J., Walters, C.E., Pryce, G., Kanuga, N., Beraud, E., Baker, D., and Adamson, P. (2003). Lovastatin inhibits brain endothelial cell Rho-mediated lymphocyte migration and attenuates experimental autoimmune encephalomyelitis. The FASEB Journal $17,905-907$.

21. Paintlia, A.S., Paintlia, M.K., Singh, A.K., Stanislaus, R., Gillg, A.G., Barbosa, E., and Singh, I. (2004). Regulation of gene expression associated with acute experimental autoimmune encephalomyelitis by Lovastatin. Journal of Neuroscience Research 77, 63-81.

22. Soma, M.R., Baetta, R., De Renzis, M.R., Mazzini, G., Davegna, C., Magrassi, L., Butti, G., Pezzotta, S., Paoletti, R., and Fumagalli, R. (1995). In vivo enhanced antitumor activity of carmustine [N, N'-bis (2-chloroethyl)-N-nitrosourea] by simvastatin. Cancer Res 55, 597-602.

23. Lu, D., Qu, C., Goussev, A., Jiang, H., Lu, C., Schallert, T., Mahmood, A., Chen, J., Li, Y., and Chopp, M. (2007). Statins increase neurogenesis in the dentate gyrus, reduce delayed neuronal death in the hippocampal CA3 region, and improve spatial learning in rat after traumatic brain injury. J Neurotraum 24, 1132-1146.

24. Lu, D., Qu, C., Goussev, A., Jiang, H., Lu, C., T, S., Mahmood, A., Chen, J., Li, Y., and Chopp, M. (2004). Atorvastatin reduces neurological deficit and increases synaptogenesis, angiogenesis, and neuronal survival in rats subjected to traumatic brain injury. J Neurotraum 21, 21-32. 
25. Wang, H., Lynch, J.R., Song, P., Yang, H.J., Yates, R.B., Mace, B., Warner, D.S., Guyton, J.R., and Laskowitz, D.T. (2007). Simvastatin and atorvastatin improve behavioral outcome, reduce hippocampal degeneration, and improve cerebral blood flow after experimental traumatic brain injury. Exp Neurol 206, 59-69.

26. Chen, S.F., Hung, T.H., Chen, C.C., Lin, K.H., Huang, Y.N., Tsai, H.C., and Wang, J.Y. (2007). Lovastatin improves histological and functional outcomes and reduces inflammation after experimental traumatic brain injury. Life Sci 81, 288-298.

27. Narayan, R.K., Michel, M.E., Ansell, B., Baethmann, A., Biegon, A., Bracken, M.B., Bullock, M.R., Choi, S.C., Clifton, G.L., Contant, C.F., Coplin, W.M., Dietrich, W.D., and et, a.I. (2002). Clinical trials in head injury. J Neurotraum 19, 503-557.

28. Sánchez-Aguilar, M., Tapia-Pérez, J.H., Sánchez-Rodríguez, J.J., Viñas-Ríos, J.M., Martínez-Pérez, P., de la Cruz-Mendoza, E., Sánchez-Reyna, M., Torres-Corzo, J.D., and Gordillo-Moscoso, A. (2013). Effect of rosuvastatin on cytokines after traumatic head injury. J Neurosurg 118, 669-675.

29. Tapia-Perez, J.H., Sanchez-Aguilar, M., Torres-Corzo, J.G., Gordillo-Moscoso, A., Martinez-Perez, P., Madeville, P., de la Cruz-Mendoza, E., and Chalita-Williams, J. (2008). Effect of Rosuvastatin on Amnesia and Disorientation after Traumatic Brain Injury (NCT00329758). J Neurotraum 25, 1011-1017.

30. Jackson, W.T., Novack, T.A., and Dowler, R.N. (1998). Effective serial measurement of cognitive orientation in rehabilitation. The Orientation Log. Arch Phys Med Rehab 79, 718-720.

31. Steiner, J.F., and Prochazka, A.V. (1997). The assessment of refill compliance using pharmacy records: methods, validity, and applications. J Clin Epidemiol 50, 105-116.

32. Austin, P.C. (2009). Balance diagnostics for comparing the distribution of baseline covariates between treatment groups in propensity-score matched samples. Stat Med 28, 3083-3107. 
33. Normand, S.L.T., Landrum, M.B., Guadagnoli, E., Ayanian, J.Z., Ryan, T.J., Cleary, P.D., and McNeil, B.J. (2001). Validating recommendations for coronary angiography following acute myocardial infarction in the elderly: A matched analysis using propensity scores. J Clin Epidemiol 54, 387-398.

34. Ho, D.E., Imai, K., King, G., and Stuart, E.A. (2007). Matching as nonparametric preprocessing for reducing model dependence in parametric causal inference. Polit Anal $15,199-236$.

35. Rosenbaum, P.R., and Rubin, D.B. (1984). Reducing bias in observational studies using subclassification on the propensity score. J Amer Stat Assoc 79, 516-524. 


\section{Table Legends}

Table 1: Table 1 lists the proportion of participants in the full sample who were positive for each covariate, in the groups receiving and not receiving statins. Low frequency comorbidities were collapsed into larger categories where noted.

Table 2: Table 2 assesses the comparability between the statin users and non-users in the full sample (left side) and in the 49 pairs of participants matched on propensity scores (right side).

Table 3: Table 3 displays early and longer-term functional outcomes for the statin users and non-users, as well as the difference between them.

Table 4: Table 4 displays early and longer-term functional outcomes for the full sample of statin users, according to whether they had sufficient statin supplies to be adherent at the time of injury (left side) or not (right side) along with the differences between them. 
Table 1: Coding and Distribution of Covariates in Full Sample

\begin{tabular}{|c|c|c|}
\hline Covariate & $\begin{array}{l}\text { Statin: Yes } \\
\qquad(N=69)\end{array}$ & $\begin{array}{l}\text { Statin: No } \\
(N=155)\end{array}$ \\
\hline Any Pre-Injury Limitations $\ddagger$ & $27(39.7 \%)$ & $46(29.7 \%)$ \\
\hline Blindness/Deafness Condition & $10(14.7 \%)$ & $13(8.4 \%)$ \\
\hline Physical Activity Limitation & $15(22.1 \%)$ & $26(16.8 \%)$ \\
\hline Learning Limitation & $12(17.6 \%)$ & $18(11.6 \%)$ \\
\hline Dressing Limitation & $9(13.2 \%)$ & $5(3.2 \%)$ \\
\hline Going Outside of Home Limitation & $9(13.2 \%)$ & $12(7.7 \%)$ \\
\hline Working Limitation & $10(14.7 \%)$ & $22(14.2 \%)$ \\
\hline Other Health Conditions $¥ \ddagger$ & $37(53.6 \%)$ & $36(23.4 \%)$ \\
\hline COPD & $7(10.1 \%)$ & $12(7.8 \%)$ \\
\hline Liver Disease & $4(5.8 \%)$ & $10(6.5 \%)$ \\
\hline Diabetes & $31(44.9 \%)$ & $18(11.7 \%)$ \\
\hline 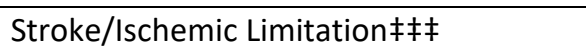 & $3(4.3 \%)$ & $12(7.7 \%)$ \\
\hline Stroke Limits Function & $3(4.3 \%)$ & $11(7.1 \%)$ \\
\hline Ischemic Limits Function & $0(0.0 \%)$ & $1(0.6 \%)$ \\
\hline Congestive Heart Failure & $8(11.6 \%)$ & $7(4.5 \%)$ \\
\hline 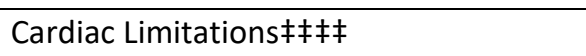 & $7(10.1 \%)$ & $6(3.9 \%)$ \\
\hline Heart Attack Limits Function & $4(5.8 \%)$ & $3(1.9 \%)$ \\
\hline Atrial Fibrillation Limits Function & $1(1.4 \%)$ & $0(0.0 \%)$ \\
\hline Angioplasty Limits Function & $0(0.0 \%)$ & $0(0.0 \%)$ \\
\hline Bypass Limits Function & $1(1.4 \%)$ & $0(0.0 \%)$ \\
\hline Pacemaker Limits Function & $1(1.4 \%)$ & $1(0.6 \%)$ \\
\hline Other Cardiac Disease Limits Function & $2(2.9 \%)$ & $1(0.6 \%)$ \\
\hline
\end{tabular}




\begin{tabular}{lcc} 
Endarterectomy Limits Function & $0(0.0 \%)$ & $0(0.0 \%)$ \\
PVD Limb Limits Function & $0(0.0 \%)$ & $0(0.0 \%)$ \\
Revascularization Limits Function & $1(1.4 \%)$ & $0(0.0 \%)$ \\
Claudication & $0(0.0 \%)$ & $1(0.6 \%)$ \\
\hline & Nominal variables are reported as $N(\%)$
\end{tabular}

¥ Any history of pre-injury limitation (deaf/blind, physical activity, learning, dressing, going outside of home, or working)

$\ddagger \ddagger$ Other health conditions (COPD, liver disease, or diabetes)

$\ddagger \ddagger \ddagger$ Stroke/Ischemic limitation (stroke or ischemic stroke that limits function)

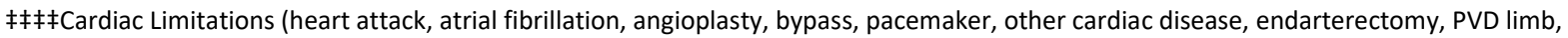
revascularization, or claudication that limits function) 
Table 2: Comparison of Statin Users and Non-Users in the Unmatched Sample and in the Propensity Score Matched Sample

Unmatched $(N=224) \quad$ Matched $(N=98)$

\begin{tabular}{|c|c|c|c|c|c|c|c|c|c|c|c|}
\hline & Statin: Yes & Statin: No & & Std. & & Var. & Statin: Yes & Statin: No & & Std. & Var. \\
\hline Covariate & $(N=69)$ & $(N=155)$ & $p$-value & Diffs & & Ratio & $(N=49)$ & $(N=49)$ & $p$-value & Diffs & Ratio \\
\hline Age at Injury (+ Age $\left.^{2}\right)$ & $68.0 \pm 10.1$ & $61.0 \pm 9.2$ & $<0.0001$ & 0.730 & †† & 1.202 & $66.1 \pm 9.8$ & $65.8 \pm 9.9$ & 0.8703 & 0.033 & (1.010) \\
\hline $\mathrm{BMI}\left(+\mathrm{BMI}^{2}\right)$ & $27.9 \pm 5.2$ & $26.8 \pm 5.8$ & 0.1878 & 0.182 & $\dagger$ & $(1.221)$ & $27.4 \pm 5.3$ & $27.7 \pm 5.1$ & 0.7773 & -0.057 & 1.078 \\
\hline Female & 27 (39.1\%) & $46(29.7 \%)$ & 0.1634 & 0.200 & †+ & & $18(36.7 \%)$ & $21(42.9 \%)$ & 0.5358 & -0.125 & $\dagger$ \\
\hline Minority & $11(16.2 \%)$ & 32 (20.6\%) & 0.4361 & -0.115 & + & & $8(16.3 \%)$ & $8(16.3 \%)$ & $>0.9999$ & 0 & \\
\hline Not Employed & 46 (67.6\%) & 75 (48.7\%) & 0.0090 & 0.391 & $+t$ & & 29 (59.2\%) & 28 (57.1\%) & 0.8377 & 0.041 & \\
\hline$<$ HS/GED Education & $12(17.6 \%)$ & 25 (16.2\%) & 0.7945 & 0.038 & & & $8(16.3 \%)$ & $10(20.4 \%)$ & 0.6018 & -0.106 & $\dagger$ \\
\hline Cause of Injury & & & 0.0103 & & & & & & 0.8229 & & \\
\hline Fall & 48 (69.6\%) & 77 (50.0\%) & & 0.407 & $+\dagger$ & & 31 (63.3\%) & 30 (61.2\%) & & 0.042 & \\
\hline Vehicular & $16(23.2 \%)$ & $45(29.2 \%)$ & & -0.138 & + & & $13(26.5 \%)$ & $12(24.5 \%)$ & & 0.047 & \\
\hline Other (+Violence) & $5(7.2 \%)$ & $32(20.8 \%)$ & & -0.397 & †† & & $5(10.2 \%)$ & $7(14.3 \%)$ & & -0.125 & + \\
\hline Substance Problem Use & 13 (19.4\%) & 47 (30.9\%) & 0.0782 & -0.268 & $+\dagger$ & & 9 (18.4\%) & $11(22.4 \%)$ & 0.6162 & -0.101 & $\dagger$ \\
\hline Smoke Cigarettes & 17 (25.0\%) & $50(32.5 \%)$ & 0.2639 & -0.166 & $\dagger$ & & $12(24.5 \%)$ & $12(24.5 \%)$ & $>0.9999$ & 0 & \\
\hline Any Pre-Injury Limitationsł & $27(39.7 \%)$ & $46(29.7 \%)$ & 0.1418 & 0.212 & $\dagger+$ & & $15(30.6 \%)$ & $18(36.7 \%)$ & 0.5214 & -0.130 & \\
\hline Other Health Conditions $¥ \ddagger$ & 37 (53.6\%) & 36 (23.4\%) & $<0.0001$ & 0.654 & $+t$ & & 19 (38.8\%) & 20 (40.8\%) & 0.8365 & -0.042 & \\
\hline
\end{tabular}




\begin{tabular}{|c|c|c|c|c|c|c|c|c|}
\hline Stroke/Ischemic Limitation $\ddagger \ddagger \ddagger$ & $3(4.3 \%)$ & $12(7.7 \%)$ & 0.3481 & -0.143 & $\dagger$ & $3(6.1 \%)$ & $3(6.1 \%)$ & $>0.9999$ \\
\hline 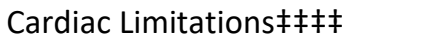 & $7(10.1 \%)$ & $6(3.9 \%)$ & 0.0637 & 0.248 & $\dagger+$ & $4(8.2 \%)$ & $3(6.1 \%)$ & 0.6949 \\
\hline CHF (any) & $8(11.6 \%)$ & $7(4.5 \%)$ & 0.0504 & 0.262 & $\dagger+$ & $4(8.2 \%)$ & $3(6.1 \%)$ & 0.6949 \\
\hline
\end{tabular}

Continuous variables are reported as mean \pm standard deviation; Nominal variables are reported as $\mathrm{N}(\%)$

+ indicates moderate imbalance $(0.1 \leq \mid$ standardized difference $\mid<0.2$

$\dagger+$ indicates substantial imbalance (|standardized difference $\mid \geq 0.2)$

‡ Any history of pre-injury limitation (deaf/blind, physical activity, learning, dressing, going outside of home, or working)

¥Other health conditions (COPD, liver disease, or diabetes)

$¥ \ddagger \ddagger S$ troke/Ischemic limitation (stroke or ischemic stroke that limits function)

$\ddagger \ddagger \ddagger$ Cardiac Limitations (heart attack, atrial fibrillation, angioplasty, bypass, pacemaker, other cardiac disease, endarterectomy, PVD limb, revascularization, or claudication that limits function) 
Table 3: Comparison of Early and Later Functional Outcomes between Statin Users and Non-Users

\begin{tabular}{|c|c|c|c|c|c|c|c|c|}
\hline \multirow[b]{2}{*}{ Outcome Variable } & \multirow[b]{2}{*}{$N$ pairs } & \multicolumn{2}{|c|}{ Statin: Yes } & \multicolumn{2}{|c|}{ Statin: No } & \multicolumn{3}{|c|}{ Difference } \\
\hline & & Mean (SD) & Median (IQR) & Mean (SD) & Median (IQR) & Mean (SD) & Median (IQR) & $p$-value ${ }^{\S}$ \\
\hline Post Traumatic Amnesia (days) & 47 & $16.9(21.3)$ & $9(2,25)$ & $18.6(21.8)$ & $12(2,25)$ & $-1.7(35.2)$ & $0(20,22)$ & $(0.8996)$ \\
\hline Time to Follow Commands (days) & 49 & $2.2(5.3)$ & $0.5(0.5,2.0)$ & $2.3(5.6)$ & $0.5(0.5,2.0)$ & $-0.2(7.9)$ & $0(-0.5,0.5)$ & $(0.8409)$ \\
\hline GCS Total & 28 & $13.6(2.8)$ & $15(14,15)$ & $13.1(2.7)$ & $14(13,15)$ & $0.6(4.3)$ & $0.5(-1,2)$ & (0.2449) \\
\hline GCS Motor & 35 & $5.7(1.0)$ & $6(6,6)$ & $5.7(0.9)$ & $6(6,6)$ & $0(1.5)$ & $0(0,0)$ & (0.9999) \\
\hline LOS Acute (days) & 49 & $14.1(10.8)$ & $12(6,18)$ & $14.0(10.5)$ & $12(7,17)$ & $0.04(16.8)$ & $0(-10,7)$ & 0.9865 \\
\hline LOS Rehab (days) & 49 & $23.2(14.0)$ & $19(14,28)$ & $20.9(12.9)$ & $18(13,22)$ & $2.2(18.7)$ & $2(-7,9)$ & $(0.3197)$ \\
\hline LOS Total (days) & 49 & $36.8(22.1)$ & $30(23,46)$ & $34.7(18.8)$ & $28(23,41)$ & $2.0(30.0)$ & $0(-14,14)$ & $(0.6244)$ \\
\hline FIM Motor Admission & 49 & $35.8(15.4)$ & $35(22,47)$ & $33.3(14.6)$ & $30(20,45)$ & $2.5(20.6)$ & $5(-14,16)$ & 0.3975 \\
\hline FIM Cognitive Admission & 49 & $15.7(6.8)$ & $16(9,20)$ & $14.4(6.3)$ & $14(9,20)$ & $1.3(9.2)$ & $1(-3,8)$ & 0.3236 \\
\hline FIM Motor Discharge & 48 & $60.6(18.8)$ & $63(51,74)$ & $63.6(15.7)$ & $65.5(52,74.5)$ & $-3.0(23.7)$ & $-2(-17.5,12.5)$ & 0.3811 \\
\hline FIM Cognitive Discharge & 49 & $23.4(5.9)$ & $23(20,28)$ & $22.4(6.1)$ & $23(18,26)$ & $1.0(7.4)$ & $1(-3,6)$ & 0.3584 \\
\hline FIM Motor 1 Year & 37 & $77.8(21.4)$ & $88(80,91)$ & $84.1(8.0)$ & $86(81,90)$ & $-6.2(20.6)$ & $0(-6,6)$ & $(0.5033)$ \\
\hline FIM Cognitive 1 Year & 37 & $30.1(6.8)$ & $33(30,34)$ & $30.9(3.4)$ & $32(29,34)$ & $-0.8(7.7)$ & $0(-1,3)$ & $(0.8692)$ \\
\hline
\end{tabular}


$\S p$-values based on paired $t$-test assuming normally distributed differences; non-normally distributed differences compared with Wilcoxon signed-rank test with corresponding $p$-values reported in parentheses 
Table 4: Comparison of outcomes between "Adherent" and "Non-Adherent" Statin Users

\begin{tabular}{|c|c|c|c|c|c|c|c|c|}
\hline \multirow[b]{2}{*}{ Outcome Variable } & \multicolumn{3}{|c|}{ Adherent } & \multicolumn{3}{|c|}{ Non-Adherent } & \multicolumn{2}{|c|}{ Difference } \\
\hline & $N$ & Mean (SD) & Median (IQR) & $N$ & Mean (SD) & Median (IQR) & Mean (SD) & $p$-value ${ }^{\S}$ \\
\hline Post Traumatic Amnesia (days) & 54 & $15.0(21.3)$ & $8(2,21)$ & 15 & $18.2(17.4)$ & $21(1,29)$ & $3.2(20.6)$ & $(0.4610)$ \\
\hline Time to Follow Commands (days) & 54 & $2.2(5.5)$ & $0.5(0.5,1.0)$ & 15 & $1.5(2.8)$ & $0.5(0.5,1.0)$ & $-0.7(5.0)$ & (0.1929) \\
\hline GCS Total & 42 & $14.2(1.9)$ & $15(14,15)$ & 12 & $13.8(2.7)$ & $15(14,15)$ & $-0.4(2.1)$ & $(0.7670)$ \\
\hline GCS Motor & 47 & $5.6(1.2)$ & $6(6,6)$ & 13 & $5.8(0.6)$ & $6(6,6)$ & $-0.2(1.1)$ & $(0.6065)$ \\
\hline LOS Acute (days) & 54 & $12.6(9.9)$ & $8(6,17)$ & 15 & $15.9(12.0)$ & $16(6,24)$ & $3.3(10.4)$ & $(0.4438)$ \\
\hline LOS Rehab (days) & 54 & $21.9(13.6)$ & $20(13,26)$ & 15 & $25.6(15.5)$ & $19(14,40)$ & $3.7(14.0)$ & $(0.4843)$ \\
\hline LOS Total (days) & 54 & $34.1(21.2)$ & $28.5(20,41)$ & 15 & $40.4(23.0)$ & $44(25,51)$ & $6.3(21.6)$ & $(0.2269)$ \\
\hline FIM Motor Admission & 54 & $35.6(15.5)$ & $34.5(22,49)$ & 14 & $31.6(16.2)$ & $30(16,47)$ & $-4.0(15.6)$ & 0.4006 \\
\hline FIM Cognitive Admission & 54 & $16.0(6.5)$ & $16.5(11,20)$ & 15 & $14.3(7.4)$ & $13(8,21)$ & $-1.8(6.7)$ & 0.3728 \\
\hline FIM Motor Discharge & 54 & $60.9(17.5)$ & $62.5(52,71)$ & 14 & $58.4(16.3)$ & $59.5(44,67)$ & $-2.5(17.3)$ & 0.6340 \\
\hline FIM Cognitive Discharge & 54 & $23.7(5.8)$ & $23.5(21,28)$ & 15 & $21.0(4.9)$ & $21(16,25)$ & $-2.7(5.6)$ & 0.0994 \\
\hline FIM Motor 1 Year & 44 & $77.7(19.1)$ & $86.5(72,91)$ & 13 & $78.6(19.0)$ & $85.0(79,89)$ & $0.9(19.1)$ & $(0.7806)$ \\
\hline FIM Cognitive 1 Year & 45 & 30.5 (5.7) & $32(29,34)$ & 13 & $28.5(6.5)$ & $30.0(24,33)$ & $-2.0(5.9)$ & $(0.2114)$ \\
\hline
\end{tabular}

$\mathrm{SD}$ = standard deviation; $\mathrm{IQR}$ = interquartile range 
$\S p$-values based on two-sample $t$-test assuming normality and equal variance; non-normally distributed differences compared with Wilcoxon rank-sum test with corresponding $p$-values reported in parentheses 


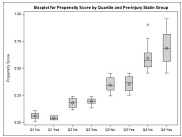




\section{Figure Legend}

Figure 1: In the Figure, subjects in both study groups ("Yes" = statin group) are divided into quartiles by propensity score. The median and interquartile range of the propensity scores for each quartile are indicated by the central line and upper and lower borders of the boxes, the mean by the diamonds, and the full range of scores by the whiskers. The figure demonstrates good comparability between groups for the first 3 quartiles and moderate imbalance for the highest quartile. 
Figure 1: Comparison of Propensity Scores for Statin Use

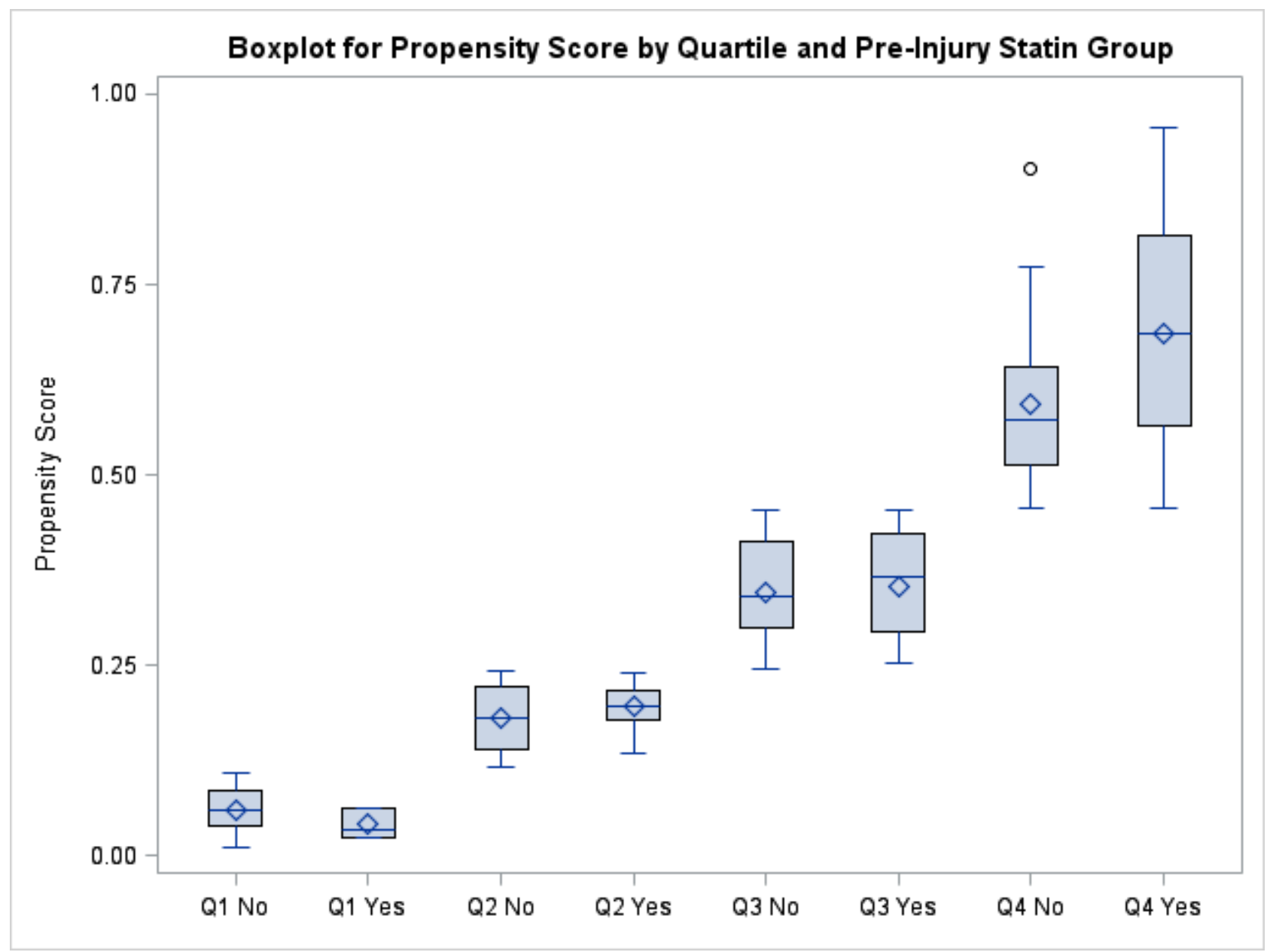

